\title{
Transmuted Generalized Gompertz distribution with application
}

\author{
Muhammad Shuaib Khan* \\ School of Mathematical and Physical Sciences, University of Newcastle \\ Callaghan, NSW 2308, Australia \\ Muhammad.S.Khan@newcastle.edu.au \\ Robert King \\ School of Mathematical and Physical Sciences, University of Newcastle \\ Callaghan, NSW 2308, Australia \\ robert.king@newcastle.edu.au \\ Irene Lena Hudson \\ School of Mathematical and Physical Sciences, University of Newcastle \\ Callaghan, NSW 2308, Australia \\ Irene.Hudson@newcastle.edu.au
}

Received 18 September 2015

Accepted 9 February 2016

\begin{abstract}
This paper introduces the four parameter transmuted generalized Gompertz distribution which includes the transmuted Gompertz, transmuted generalized exponential, transmuted exponential, Gompertz, generalized exponential and exponential distributions as special cases and studies its statistical properties. Explicit expressions are derived for the quantile, moments, moment generating function and entropies. Maximum likelihood estimation is used to estimate the model parameters. Finally, two applications of the new distribution is illustrated using reliability data sets.

Keywords: Reliability functions; moment estimation; maximum likelihood estimation.
\end{abstract}

2000 Mathematics Subject Classification: 90B25, 62N05

\section{Introduction}

In recent years, many different method have been proposed for adding parameters to the classical distributions for obtaining more flexible new family of lifetime distributions. There has been a renewed interest in developing the transmuted family of lifetime distributions for modelling lifetime data. A significant amount of work has been attributed towards developing the transmuted family that allows extra flexibility for explaining the bathtub shaped curve. The transmuted generated method pioneered by Shaw and Buckley

* Corresponding author. 
(2007) has been applied extensively to construct new distributions. Many different areas of the real world applications from the engineering, medical sciences, environmental sciences, areanoutics, hyderology, agriculture, pharmacutical sciences, psychology, metrology, economics and actuarial sciences among others have shown that the classical distributions does not fit the data very well. Since there is clear needs of extended distributions that can provide better estimates for real world situations. The main aim in this paper is to provide another extension of the generalized gompertz distribution using the transmuted-G generator defined by Bourguignon et al (2016). The Gompertz distribution was pioneered to modelling human mortality data by Gompertz (1825). The Gompertz distribution has wide applications in demography, actuarial and biological sciences. The maximum likelihood estimates for the parameters of the Gompertz distributional was studied by Garg et al. (1970). Recently Gohary et al. (2011) proposed the generalized Gompertz distribution and compare this model with exponential family of lifetime distributions, which has the following cdf as

$$
G(x ; \alpha, \beta, \eta)=\left[1-\exp \left\{-\frac{\alpha}{\eta}\left(e^{\eta x}-1\right)\right\}\right]^{\beta}, \quad(x>0),
$$

The probability density function corresponding to (1) is given by

$$
g(x ; \alpha, \beta, \eta)=\alpha \beta e^{\eta x} \exp \left\{-\frac{\alpha}{\eta}\left(e^{\eta x}-1\right)\right\}\left[1-\exp \left\{-\frac{\alpha}{\eta}\left(e^{\eta x}-1\right)\right\}\right]^{\beta-1},
$$

respectively. where $\alpha, \eta>0$ are the scale parameters and $\beta$ is the shape parameter.

Recently sevaral distributions have been proposed from the transmuted family of lifetime distributions. Aryal et al. (2011) proposed the transmuted Weibull distribution with applications. Merovci (2013) studied the transmuted Rayleigh distribution with an application to bladder cancer data. More recently Khan and King (2014a, 2014b) proposed the transmuted Inverse Weibull distribution and discussed theoretical properties of this distribution with application to lifetime data. Khan et al. (2014c) proposed the transmuted Gompertz distribution and studied various structural properties with an application to reliability data. Moniem and Seham (2015) studied the transmuted Gompertz distribution and discussed some mathematical results. Khan et al. (2013) proposed the transmuted Chen distribution and discussed theoretical properties with application. Yuzhu et al. (2014) proposed the transmuted linear exponential distribution and compare its goodness of fit with the twelve different lifetime distributions. The motivation of this study is to introduce new model which has the ability to describe bathtub shaped failure rate function.

By using the quadratic rank transmutation map technique proposed by Shaw and Buckley (2007), we develop the four parameter transmuted generalized Gompertz distribution. According to this approach a random variable $\mathrm{X}$ is said to have a transmuted distribution if its cumulative distribution function (cdf) satisfies the following relationship

$$
F(x)=(1+\lambda) G(x)-\lambda G(x)^{2}, \quad|\lambda| \leq 1
$$

and

$$
f(x)=g(x)\{(1+\lambda)-2 \lambda G(x)\},
$$

where $G(x)$ is the cdf of the base distribution, $g(x)$ and $f(x)$ are the corresponding probability density functions (pdf) associated with $G(x)$ and $F(x)$, respectively. It is important to note that at $\lambda=0$ we have the distribution of the base random variable. The paper is organised as follows, in Section 2, we present the analytical shapes of the probability density and hazard functions of the four parameter transmuted generalized Gompertz distribution. We formulate expressions for the moments and entropies in Section 3. Maximum likelihood estimates (MLEs) of the unknown parameters are discussed in Section 4. Simulation study is performed in Section 5. The usefulness of the proposed model is illustrated in Section 6. Concluding remarks are addressed in Section 7. 


\section{Transmuted generalized Gompertz distribution}

A random variable $X$ having the transmuted generalized Gompertz cumulative distribution function (cdf) and it is defined through the QRTM technique as

$$
F(x ; \alpha, \beta, \eta, \lambda)=\left[1-\exp \left\{-\frac{\alpha}{\eta}\left(e^{\eta x}-1\right)\right\}\right]^{\beta}\left\{1+\lambda-\lambda\left[1-\exp \left\{-\frac{\alpha}{\eta}\left(e^{\eta x}-1\right)\right\}\right]^{\beta}\right\},
$$

The pdf corresponding to (5) is given by

$$
\begin{aligned}
f(x ; \alpha, \beta, \eta, \lambda)=\alpha \beta e^{\eta x} \exp \left\{-\frac{\alpha}{\eta}\left(e^{\eta x}-1\right)\right\} & {\left[1-\exp \left\{-\frac{\alpha}{\eta}\left(e^{\eta x}-1\right)\right\}\right]^{\beta-1} } \\
\times & \left\{1+\lambda-2 \lambda\left[1-\exp \left\{-\frac{\alpha}{\eta}\left(e^{\eta x}-1\right)\right\}\right]^{\beta}\right\},
\end{aligned}
$$

$\alpha=1, \eta=0.5$
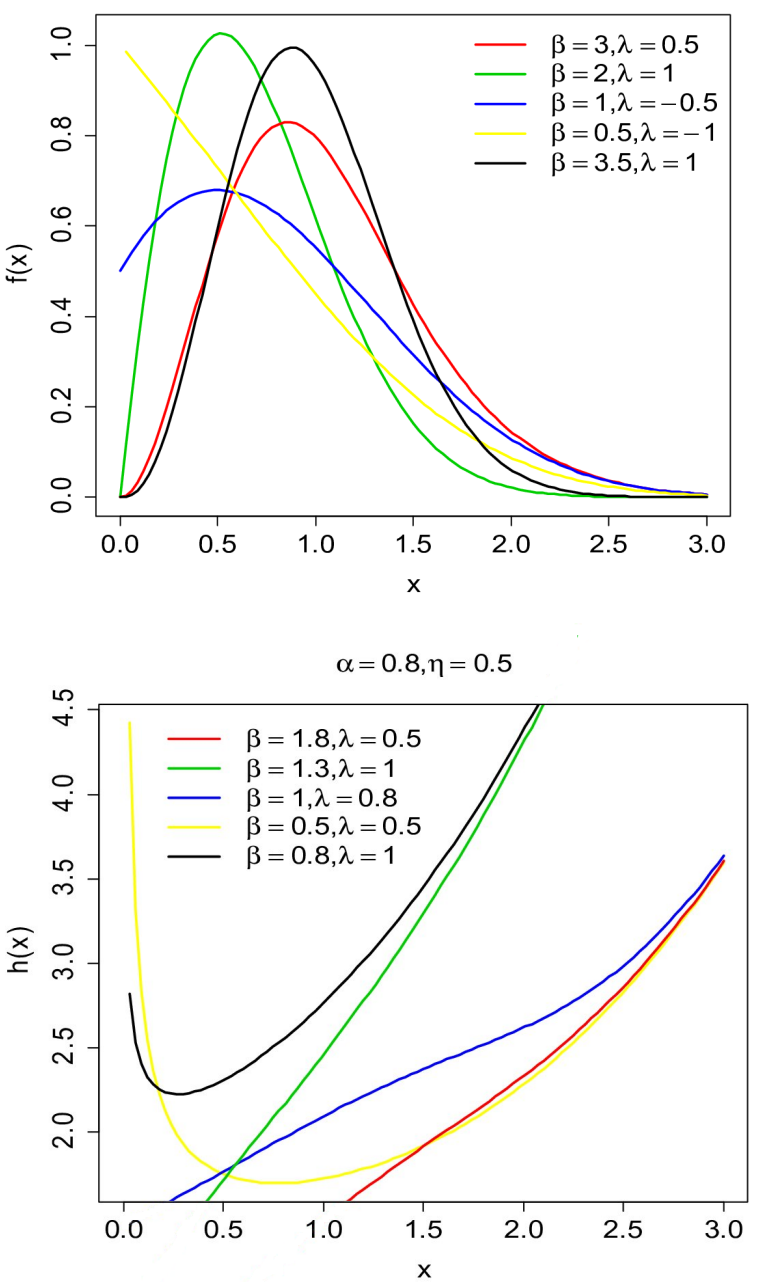



$\alpha=0.5, \eta=0.5, \beta=0.5$

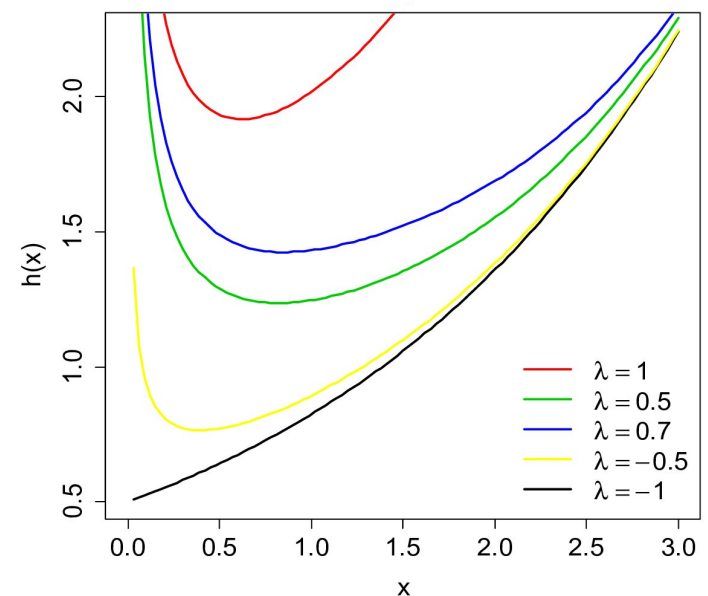

Fig. 1. Plots of the TGG density and hazard functions. 
where $\alpha, \beta, \eta>0, \lambda \leq 1$ and $x>0$. The parameter $\alpha$ controls the scale of the distribution whereas the parameter $\beta$ controls its shape. The parameter $\eta$ controls the accelerating factor whereas the parameter $\lambda$ is the transmuted parameter which provides an extra flexibility for fitting lifetime data. A random variable $X$ having the transmuted generalized Gompertz distribution and denoted as $X \sim T G G(x ; \alpha, \beta, \eta, \lambda)$. If $\lambda=0$ the TGG distribution reduces to the classical GG distribution. The transmuted Gompertz distribution is a special case of the proposed model when the shape parameter $\beta=1$. Further in addition to $\lambda=0$ and $\beta=1$, Equation (6) reduces to the Gompertz distribution. The quantile function corresponding to (6) is given by

$$
Q(u)=\frac{1}{\eta} \ln \left[1+\ln \left\{1-\frac{\eta}{\alpha} \ln \left(1-\left(\frac{(1+\lambda)-\sqrt{(1+\lambda)^{2}-4 \lambda u}}{2 \lambda}\right)^{\frac{1}{\beta}}\right)\right\},\right.
$$

The hazard function is given by

$$
h(x ; \alpha, \beta, \eta, \lambda)=\frac{\left\{\begin{array}{c}
\left.\alpha \beta e^{\eta x} \exp \left\{-\frac{\alpha}{\eta}\left(e^{\eta x}-1\right)\right\}\left[1-\exp \left\{-\frac{\alpha}{\eta}\left(e^{\eta x}-1\right)\right\}\right]^{\beta-1}\right\} \\
\times\left\{1+\lambda-2 \lambda\left[1-\exp \left\{-\frac{\alpha}{\eta}\left(e^{\eta x}-1\right)\right\}\right]^{\beta}\right\}
\end{array}\right\}}{1-\left[1-\exp \left\{-\frac{\alpha}{\eta}\left(e^{\eta x}-1\right)\right\}\right]^{\beta}\left\{1+\lambda-\lambda\left[1-\exp \left\{-\frac{\alpha}{\eta}\left(e^{\eta x}-1\right)\right\}\right]^{\beta}\right\}} .
$$

Figure 1 provides some plots of the TGG density and hazard curves for some selected choice of parameters and showing that it is quite flexible for modelling lifetime data. We have supporting evidence from Figure 1 that the proposed model has increasing and decreasing hazard functions for some selected parameter values.

\section{Moments and Entropy}

This section address the $k^{\text {th }}$ moment, moment generating function, the Rényi and q-entropies and visualize the skewness and kurtosis measures for the transmuted generalized Gompertz distribution.

Theorem 1. If $X$ has the $\operatorname{TGG}(x ; \alpha, \beta, \eta, \lambda)$ distribution with $|\lambda| \leq 1$, then the $k^{\text {th }}$ moment of $X$ say $\dot{\mu}_{k}$ is given as follows

$$
\dot{\mu}_{k}=\left\{(1+\lambda) \sum_{i, j, h=0}^{\infty} \mathcal{v}_{i, j, k}\left(\begin{array}{c}
\beta-1 \\
i
\end{array}\right)-2 \lambda \sum_{i, j, k=0}^{\infty} \mathcal{v}_{i, j, k}\left(\begin{array}{c}
2 \beta-1 \\
i
\end{array}\right)\right\} \Gamma(k+1) .
$$

Proof. By definition the $k^{\text {th }}$ moment of $X$ of the TGG distribution as follows

$$
\begin{aligned}
\dot{\mu}_{k}=(1+\lambda) \int_{0}^{\infty} x^{k} \alpha \beta e^{\eta x} \exp \{ & \left.-\frac{\alpha}{\eta}\left(e^{\eta x}-1\right)\right\}\left[1-\exp \left\{-\frac{\alpha}{\eta}\left(e^{\eta x}-1\right)\right\}\right]^{\beta-1} d x \\
& -2 \lambda \int_{0}^{\infty} x^{k} \alpha \beta e^{\eta x} \exp \left\{-\frac{\alpha}{\eta}\left(e^{\eta x}-1\right)\right\}\left[1-\exp \left\{-\frac{\alpha}{\eta}\left(e^{\eta x}-1\right)\right\}\right]^{2 \beta-1} d x,
\end{aligned}
$$

Using the series expansion, we obtain 


$$
\begin{aligned}
\mu_{k}=(1+\lambda) \sum_{i, j=0}^{\infty} \frac{\alpha^{j+1} \beta(-1)^{i+j}(i+1)^{j}}{\eta^{j} \exp \{-\alpha(i+1) / \eta\}}\left(\begin{array}{c}
\beta-1 \\
i
\end{array}\right) \int_{0}^{\infty} x^{k} \exp \{\eta x(j+1)\} d x \\
-2 \lambda \sum_{i, j=0}^{\infty} \frac{\alpha^{j+1} \beta(-1)^{i+j}(i+1)^{j}}{\eta^{j} \exp \{-\alpha(i+1) / \eta\}}\left(\begin{array}{c}
2 \beta-1 \\
i
\end{array}\right) \int_{0}^{\infty} x^{k} \exp \{\eta x(j+1)\} d x .
\end{aligned}
$$

The above integral reduces to the $k^{\text {th }}$ moment as

$$
\dot{\mu}_{k}=(1+\lambda) \sum_{i, j, k=0}^{\infty} v_{i, j, k}\left(\begin{array}{c}
\beta-1 \\
i
\end{array}\right) \Gamma(k+1)-2 \lambda \sum_{i, j, k=0}^{\infty} v_{i, j, k}\left(\begin{array}{c}
2 \beta-1 \\
i
\end{array}\right) \Gamma(k+1),
$$

where

$$
v_{i, j, k}=\frac{\alpha^{j+1} \beta(-1)^{i+j+k+1}(i+1)^{j}}{\eta^{j} \exp \{-\alpha(i+1) / \eta\}\{(j+1) \eta\}^{k+1}} .
$$

The central moment $\left(\mu_{k}\right)$ and the cumulants $\left(\xi_{k}\right)$ of $X$ can be obtained from (9) as

$$
\mu_{k}=\sum_{i=0}^{k}\left(\begin{array}{c}
k \\
i
\end{array}\right)(-1)^{i} \dot{\mu}_{1}^{k} \dot{\mu}_{k-i}, \text { and } \xi_{k}=\dot{\mu}_{k}-\sum_{i=1}^{k-1}\left(\begin{array}{c}
k-1 \\
i-1
\end{array}\right) \xi_{i} \dot{\mu}_{k-i}
$$

The important features of the TGG distribution can be analysed using the $k^{t h}$ moments such as measure of central tendency, measure of dispersions, skewness and kurtosis measures.

Theorem 2. If $X$ has the $T G G(x ; \alpha, \beta, \eta, \lambda)$ distribution with $|\lambda| \leq 1$, then the moment generating function of $X$, say $M_{X}(t)$ is given as follows

$$
\begin{array}{r}
M_{X}(t)=(1+\lambda) \sum_{m=0}^{\infty} \sum_{i, j=0}^{\infty}\left(\begin{array}{c}
\beta-1 \\
i
\end{array}\right) \frac{t^{m} \alpha^{j+1} \beta(-1)^{i+j+m+1}(i+1)^{j}}{m ! \eta^{j} \exp \{-\alpha(i+1) / \eta\}\{(j+1) \eta\}^{m+1}} \Gamma(m+1) \\
\quad-2 \lambda \sum_{m=0}^{\infty} \sum_{i, j=0}^{\infty}\left(\begin{array}{c}
2 \beta-1 \\
i
\end{array}\right) \frac{t^{m} \alpha^{j+1} \beta(-1)^{i+j+m+1}(i+1)^{j}}{m ! \eta^{j} \exp \{-\alpha(i+1) / \eta\}\{(j+1) \eta\}^{m+1}} \Gamma(m+1) .
\end{array}
$$

Proof. By definition the mgf is as follows

$$
\begin{aligned}
M_{X}(t)=(1+\lambda) \int_{0}^{\infty} e^{t x} \alpha \beta e^{\eta x} \exp \left\{-\frac{\alpha}{\eta}\left(e^{\eta x}-1\right)\right\}\left[1-\exp \left\{-\frac{\alpha}{\eta}\left(e^{\eta x}-1\right)\right\}\right]^{\beta-1} d x \\
-2 \lambda \int_{0}^{\infty} e^{t x} \alpha \beta e^{\eta x} \exp \left\{-\frac{\alpha}{\eta}\left(e^{\eta x}-1\right)\right\}\left[1-\exp \left\{-\frac{\alpha}{\eta}\left(e^{\eta x}-1\right)\right\}\right]^{2 \beta-1} d x,
\end{aligned}
$$

Using the series expansion, we obtain

$$
M_{X}(t)=(1+\lambda) \sum_{m=0}^{\infty} \sum_{i, j=0}^{\infty}\left(\begin{array}{c}
\beta-1 \\
i
\end{array}\right) \frac{t^{m} \alpha^{j+1} \beta(-1)^{i+j}(i+1)^{j}}{m ! \eta^{j} \exp \{-\alpha(i+1) / \eta\}} \int_{0}^{\infty} x^{m} \exp \{\eta x(j+1)\} d x
$$




$$
-2 \lambda \sum_{m=0}^{\infty} \sum_{i, j=0}^{\infty}\left(\begin{array}{c}
2 \beta-1 \\
i
\end{array}\right) \frac{t^{m} \alpha^{j+1} \beta(-1)^{i+j}(i+1)^{j}}{m ! \eta^{j} \exp \{-\alpha(i+1) / \eta\}} \int_{0}^{\infty} x^{m} \exp \{\eta x(j+1)\} d x .
$$

The above integral reduces to the mgf of the TGG distribution as

$$
\begin{aligned}
M_{X}(t)=(1+\lambda) \sum_{m=0}^{\infty} \sum_{i, j=0}^{\infty}\left(\begin{array}{c}
\beta-1 \\
i
\end{array}\right) \frac{t^{m} \alpha^{j+1} \beta(-1)^{i+j+m+1}(i+1)^{j}}{m ! \eta^{j} \exp \{-\alpha(i+1) / \eta\}\{(j+1) \eta\}^{m+1}} \Gamma(m+1) \\
\quad-2 \lambda \sum_{m=0}^{\infty} \sum_{i, j=0}^{\infty}\left(\begin{array}{c}
2 \beta-1 \\
i
\end{array}\right) \frac{t^{m} \alpha^{j+1} \beta(-1)^{i+j+m+1}(i+1)^{j}}{m ! \eta^{j} \exp \{-\alpha(i+1) / \eta\}\{(j+1) \eta\}^{m+1}} \Gamma(m+1) .
\end{aligned}
$$

The transmuted generalized gompertz distribution allows an extra flexibility in the skewness and kurtosis than the baseline model. Figure 2 shows the behavior of the skewness and kurtosis measures of the TGG distribution as a function of the transmuted parameter $\lambda$ by using equation (7). The entropy of a random variable $X$ with density $f(x)$ is a measure of variation of the uncertainty. A large value of entropy indicates the greater uncertainty in the data. The Rényi entropy defined as

$$
I_{R}(\rho)=\frac{1}{1-\rho} \log \left\{\int f(x)^{\rho} d x\right\},
$$

where $\rho>0$ and $\rho \neq 1$. Using (6) the integral in $I_{R}(\rho)$ can be defined as

$$
\begin{gathered}
\int_{0}^{\infty} f(x)^{\rho} d x=\int_{0}^{\infty}(\alpha \beta)^{\rho} e^{\rho \eta x} \exp \left\{-\frac{\alpha \rho}{\eta}\left(e^{\eta x}-1\right)\right\}\left[1-\exp \left\{-\frac{\alpha}{\eta}\left(e^{\eta x}-1\right)\right\}\right]^{\rho(\beta-1)} \\
\times\left\{1+\lambda-2 \lambda\left[1-\exp \left\{-\frac{\alpha}{\eta}\left(e^{\eta x}-1\right)\right\}\right]^{\beta}\right\} d x, \\
\int_{0}^{\infty} f(x)^{\rho} d x=\sum_{i, j=0}^{\infty} \mathcal{T}_{i, j} \int_{0}^{\infty} \exp (\rho \eta x) \exp \{-\alpha(\rho+j)(\exp (\eta x)-1) / \eta\} d x
\end{gathered}
$$

where

$$
\mathcal{T}_{i, j}=(\alpha \beta)^{\rho}\left(\begin{array}{c}
\rho \\
i
\end{array}\right)\left(\begin{array}{c}
\rho(\beta-1)+\beta i \\
j
\end{array}\right)(-1)^{i+j}\left(\frac{2 \lambda}{1+\lambda}\right)^{i}(1+\lambda)^{\rho} .
$$

Finally the above integral reduces to

$$
\int_{0}^{\infty} f(x)^{\rho} d x=\sum_{i, j, k=0}^{\infty} \mathcal{T}_{i, j}(-1)^{k} \exp \{\alpha(\rho+j) / \eta\} \frac{(\rho+j)^{k} \alpha^{k}}{(\rho+k) \eta^{k+1}} .
$$

Therefore, the Rényi entropy of $X$ can be expressed by

$$
\begin{aligned}
I_{R}(\rho)=\frac{\rho}{1-\rho} \log (\alpha)+\frac{\rho}{1-\rho} \log (\beta)+\frac{\rho}{1-\rho} \log (1+\lambda)+\frac{1}{1-\rho} \log \\
\qquad\left\{\sum_{i, j, k=0}^{\infty}\left(\begin{array}{c}
\rho \\
i
\end{array}\right)\left(\begin{array}{c}
\rho(\beta-1)+\beta i \\
j
\end{array}\right)(-1)^{i+j+k}\left(\frac{2 \lambda}{1+\lambda}\right)^{i} \exp \{\alpha(\rho+j) / \eta\} \frac{(\rho+j)^{k} \alpha^{k}}{(\rho+k) \eta^{k+1}}\right\} .
\end{aligned}
$$


The $q$-(or $\alpha$ entropy) was introduced by Havrda and Charvat (1967) and is the one parameter generalization of the Shannon entropy. Ullah, 1996 stated that $q$-(or $\alpha$ entropy) measures the monotonic functions of the Rényi entropy and is defined as

$$
I_{H}(q)=\frac{1}{q-1}\left\{1-\int_{0}^{\infty} f(x)^{q} d x\right\}
$$

where $q>0$ and $q \neq 1$. Using (6) the integral in $I_{H}(q)$ can be defined as

$$
\begin{aligned}
\int_{0}^{\infty} f(x)^{q} d x=\int_{0}^{\infty}(\alpha \beta)^{q} e^{q \eta x} \exp \left\{-\frac{\alpha q}{\eta}\left(e^{\eta x}-1\right)\right\}\left[1-\exp \left\{-\frac{\alpha}{\eta}\left(e^{\eta x}-1\right)\right\}\right]^{q(\beta-1)} \\
\times\left\{1+\lambda-2 \lambda\left[1-\exp \left\{-\frac{\alpha}{\eta}\left(e^{\eta x}-1\right)\right\}\right]^{\beta}\right\}^{q} d x,
\end{aligned}
$$

Using (11) and (13), we obtain the expression of the $q$ - entropy as

$$
I_{H}(q)=\frac{1}{q-1}\left\{1-\sum_{i, j, k=0}^{\infty} v_{i, j}(-1)^{i+j+h}(\alpha \beta)^{q} \exp \{\alpha(\rho+j) / \eta\} \frac{(\rho+j)^{k} \alpha^{k}}{(\rho+k) \eta^{k+1}}\right\},
$$

where

$$
v_{i, j}=\left(\begin{array}{c}
q \\
i
\end{array}\right)\left(\begin{array}{c}
q(\beta-1)+\beta i \\
j
\end{array}\right)\left(\frac{2 \lambda}{1+\lambda}\right)^{i}(1+\lambda)^{q} .
$$
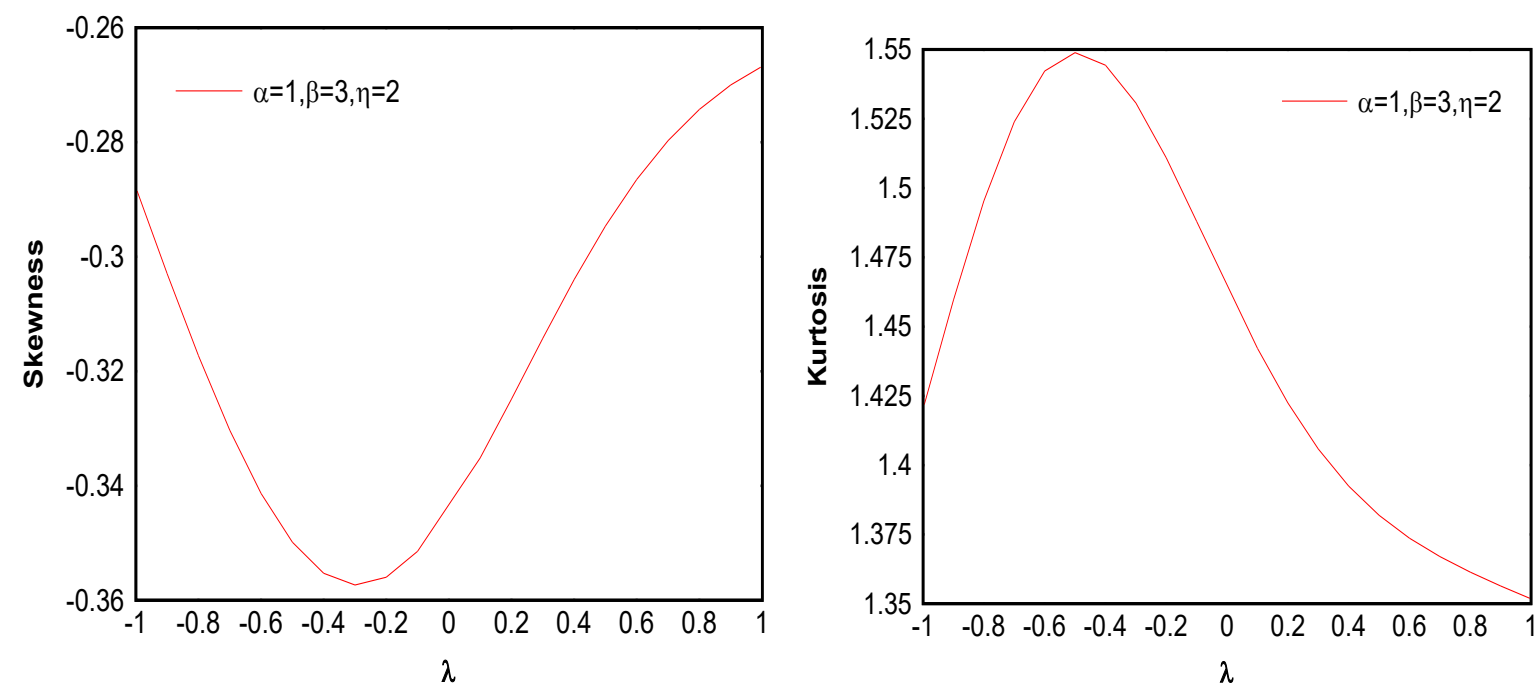

Fig. 2. Skewness and kurtosis of the TGG distribution.

\section{Maximum Likelihood Estimation}

Consider the random sample $x_{1}, x_{2}, \ldots, x_{n}$ consisting of $n$ observations from the $T G G(x ; \alpha, \beta, \eta, \lambda)$ distribution having the probability density function given in equation (6). Let $\theta=(\alpha, \beta, \eta, \lambda)^{\mathrm{T}}$ be the vector of the parameters. The log-likelihood function for $\theta$ can be expressed as 


$$
\begin{aligned}
\mathcal{L}(\theta)=n \ln \alpha+n \ln \beta-\frac{\alpha}{\eta} \sum_{i=1}^{n}\left(e^{\eta x_{i}}-1\right)+(\beta-1) \sum_{i=1}^{n} \ln \left[1-\exp \left\{-\frac{\alpha}{\eta}\left(e^{\eta x_{i}}-1\right)\right\}\right] \\
+\eta \sum_{i=1}^{n} x_{i}+\sum_{i=1}^{n} \ln \left\{1+\lambda-2 \lambda\left[1-\exp \left\{-\frac{\alpha}{\eta}\left(e^{\eta x_{i}}-1\right)\right\}\right]^{\beta}\right\}
\end{aligned}
$$

By differentiating (14) with respect to $\alpha, \beta, \eta$ and $\lambda$ then equating it to zero, we obtain the component of the score vector $U(\theta)$ are given by

$$
\begin{aligned}
& \frac{\partial \mathcal{L}(\theta)}{\partial \alpha}=\frac{n}{\alpha}-\frac{1}{\eta} \sum_{i=1}^{n}\left(e^{\eta x_{i}}-1\right)+(\beta-1) \sum_{i=1}^{n} \frac{z_{i}\left(e^{\eta x_{i}}-1\right)}{\eta\left[1-z_{i}\right]}+\frac{2 \lambda \beta}{\eta} \sum_{i=1}^{n} \frac{\left[1-z_{i}\right]^{\beta-1} z_{i}\left(e^{\eta x_{i}}-1\right)}{\left\{1+\lambda-2 \lambda\left[1-z_{i}\right]^{\beta}\right\}}, \\
& \frac{\partial \mathcal{L}(\theta)}{\partial \beta}=\frac{n}{\beta}+\sum_{i=1}^{n} \ln \left[1-z_{i}\right]-2 \lambda \sum_{i=1}^{n} \frac{\left[1-z_{i}\right]^{\beta-1} \ln \left[1-z_{i}\right]}{\left\{1+\lambda-2 \lambda\left[1-z_{i}\right]^{\beta}\right\}}, \\
& \frac{\partial \mathcal{L}(\theta)}{\partial \eta}=\sum_{i=1}^{n} x_{i}-\frac{\alpha}{\eta^{2}} \sum_{i=1}^{n}\left\{e^{\eta x_{i}}\left(\eta x_{i}-1\right)+1\right\}+\frac{\alpha(\beta-1)}{\eta^{2}} \sum_{i=1}^{n} \frac{z_{i}\left\{e^{\eta x_{i}}\left(\eta x_{i}-1\right)+1\right\}}{1-z_{i}} \\
& \frac{\partial \mathcal{L}(\theta)}{\partial \lambda}=\sum_{i=1}^{n} \frac{1-2\left[1-z_{i}\right]^{\beta}}{\left\{1+\lambda-2 \lambda\left[1-z_{i}\right]^{\beta}\right\}},
\end{aligned}
$$

where $z_{i}=\exp \left\{-\frac{\alpha}{\eta}\left(e^{\eta x_{i}}-1\right)\right\}$ respectively, the asymptotic variance covariance matrix of MLEs for the parameter vector $\theta=(\alpha, \beta, \eta, \lambda)^{\mathrm{T}}$ can be considered as the multivariate normal and its inverse of the expected information matrix is given by

$$
((\hat{\alpha}-\alpha),(\hat{\beta}-\beta),(\hat{\eta}-\eta),(\hat{\lambda}-\lambda)) \sim \mathrm{N}_{4}\left\{0, K(\theta)^{-1}\right\},
$$

where $K(\theta)^{-1}$ is the variance covariance matrix of the unknown parameters. Here $K(\hat{\theta})$ is the total observed information matrix evaluated at $\hat{\theta}$. The multivariate normal distribution can be used to obtain an approximate $100(1-\gamma) \%$ confidence intervals for the parameters $\alpha, \beta, \eta$ and $\lambda$ can be determined as

$$
\hat{\alpha} \pm Z_{\frac{\gamma}{2}} \sqrt{\widehat{V}_{11}}, \quad \hat{\beta} \pm Z_{\frac{\gamma}{2}} \sqrt{\hat{V}_{22}}, \quad \hat{\eta} \pm Z_{\frac{\gamma}{2}} \sqrt{\widehat{V}_{33}}, \quad \hat{\lambda} \pm Z_{\frac{\gamma}{2}} \sqrt{\widehat{V}_{44}}
$$

where $Z_{\frac{\gamma}{2}}$ is the upper $\gamma$ th percentile of the standard normal distribution. 


\section{Simulation}

This section evaluates the performance of MLEs by using Monte Carlo simulation. The inversion method is used to generate samples from the TGG distribution for different sample sizes $n=25,50,100,200,400$, with different choices of parameters using equation (7). The simulation process is repeated for 1000 times using the BFGS optimization method in $\mathrm{R}$ and results were displayed in Tables 1-2. We perform the simulation study to access the cost of the additional transmuted parameter. Tables 1-2 show the output of the ML estimates and standard deviation with respect to the sample sizes for each choice of the vector of the parameters. Figure 3 shows the graphical comparison of the exact TGG densities and histogram from four simulated data sets for some selected choice of parameters.

(a)

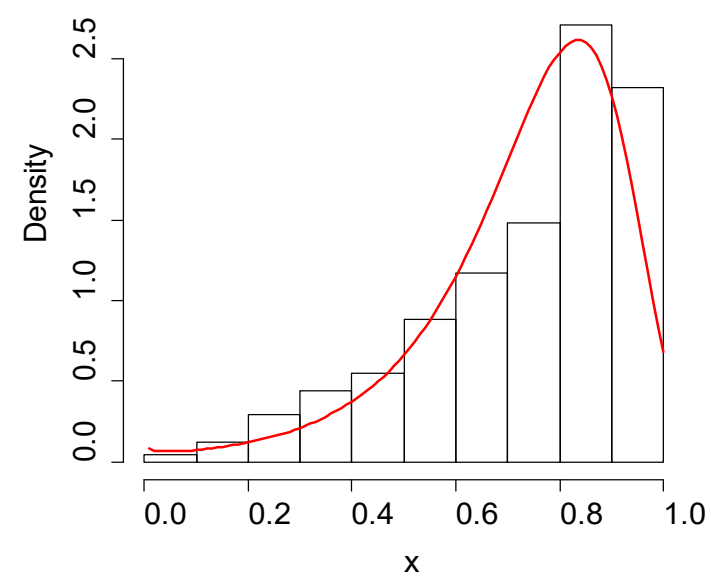

(c)

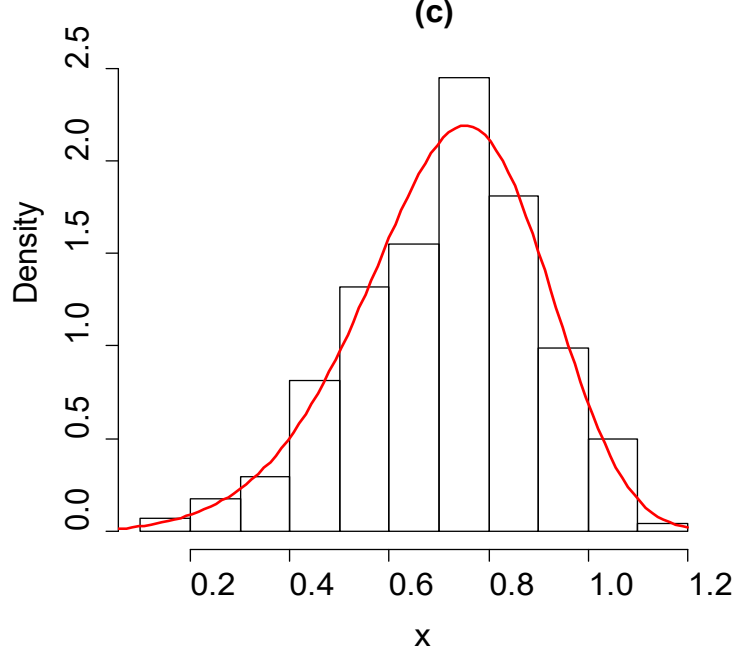

(b)

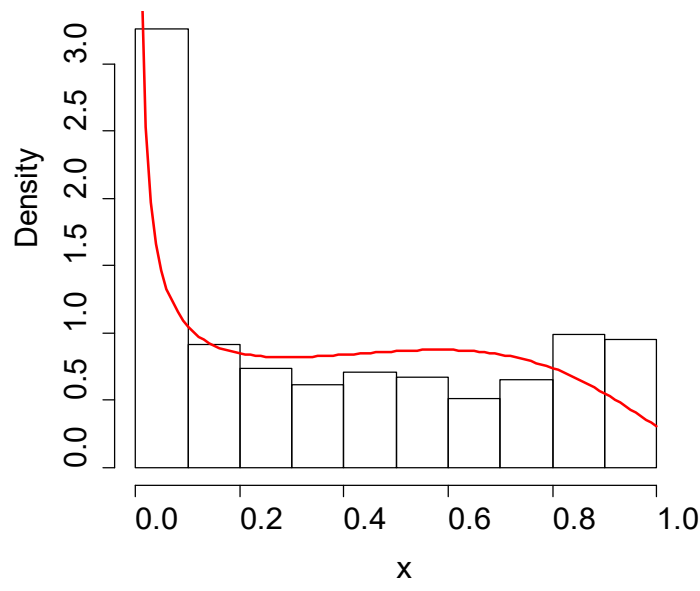

(d)

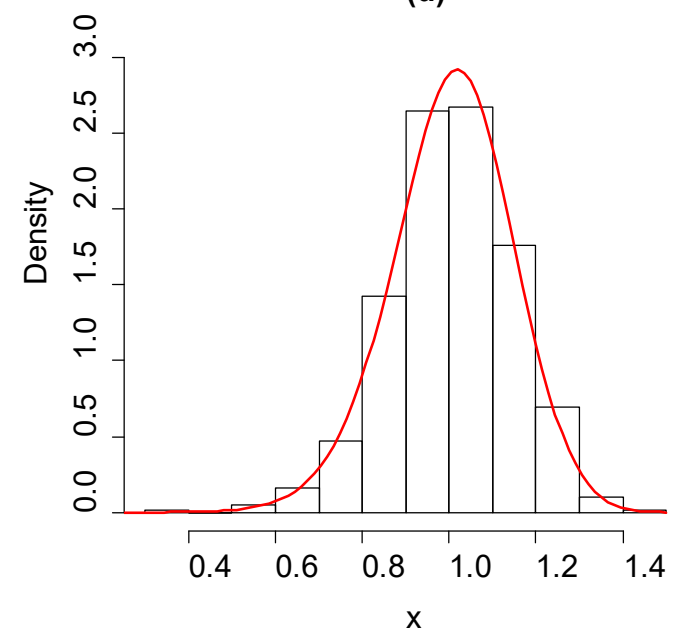

Fig. 3. Plots of the TGG densities for simulated data sets,
(a) $\alpha=1 ; \beta=1 ; \eta=0.5 ; \lambda=0.5$
(b) $\alpha=0.5 ; \beta=3 ; \eta=2 ; \lambda=0.5$
(c) $\alpha=1 ; \beta=3 ; \eta=1 ; \lambda=-0.5$
(d) $\alpha=0.5 ; \beta=3.5 ; \eta=1 ; \lambda=-1$. 
Table 1. Estimated values of $\hat{\theta}$ based on MLE

\begin{tabular}{cccccc}
\hline$\theta=(\alpha, \beta, \eta, \lambda)$ & $n$ & Estimates & & & \\
\cline { 2 - 5 } & & $\hat{\alpha}$ & $\hat{\beta}$ & $\hat{\eta}$ & $\hat{\lambda}$ \\
\hline $1,0.5,3,-1$ & 25 & 1.0333 & 1.0491 & 2.7090 & 0.1144 \\
& 50 & 0.5395 & 0.9170 & 3.5145 & 0.1121 \\
& 100 & 0.4779 & 0.8743 & 3.6058 & 0.2147 \\
& 200 & 0.6031 & 1.0473 & 3.0920 & 0.3354 \\
& 400 & 0.6170 & 0.9035 & 2.9930 & 0.1006 \\
$1,1,3,-0.5$ & 25 & 0.7552 & 1.7753 & 2.7729 & 0.2809 \\
& 50 & 0.5740 & 1.0029 & 3.4876 & 0.0381 \\
& 100 & 0.3216 & 0.9026 & 3.9366 & 0.2634 \\
& 200 & 0.4153 & 0.8829 & 3.6788 & 0.0599 \\
$1,2,3,0.5$ & 400 & 0.5390 & 1.2574 & 3.3574 & 0.2164 \\
& 25 & & & & \\
& 50 & 0.5390 & 1.2574 & 3.3574 & 0.2164 \\
& 100 & 0.7875 & 2.0276 & 2.7576 & 0.0389 \\
& 200 & 1.5082 & 1.5797 & 3.1569 & -0.0322 \\
$1,3,3,1$ & 400 & 0.6250 & 1.5061 & 3.0435 & -0.8466 \\
& & & & 3.2281 & 0.3129 \\
& 25 & 0.5667 & 2.4773 & 3.7516 & 0.4424 \\
& 50 & 0.7011 & 2.0569 & 3.6282 & 0.0001 \\
& 100 & 0.8162 & 2.9175 & 3.5197 & 0.2165 \\
& 200 & 0.8417 & 2.4954 & 3.4698 & 0.0598 \\
& 400 & 0.9734 & 2.7551 & 3.2694 & 0.0731 \\
\hline
\end{tabular}

Table 2. Standard deviations of the estimate of $\hat{\theta}$ based on MLE

\begin{tabular}{cccccc}
\hline$\theta=(\alpha, \beta, \eta, \lambda)$ & $n$ & SD & & & \\
\cline { 2 - 5 } & & $\hat{\alpha}$ & $\hat{\beta}$ & $\hat{\eta}$ & $\hat{\lambda}$ \\
\hline $1,0.5,3,-1$ & 25 & 1.2310 & 0.5367 & 1.9683 & 0.6143 \\
& 50 & 0.6200 & 0.3053 & 1.5169 & 0.7243 \\
& 100 & 0.4960 & 0.1999 & 1.2267 & 0.7059 \\
& 200 & 0.3797 & 0.1759 & 0.6934 & 0.4735 \\
& 400 & 0.2751 & 0.0983 & 0.5294 & 0.3348 \\
$1,1,3,-0.5$ & & & & & \\
& 25 & 0.5919 & 0.2929 & 1.1518 & 0.8547 \\
& 50 & 0.5866 & 0.3604 & 1.4302 & 0.5895 \\
& 100 & 0.3700 & 0.2296 & 1.2538 & 0.7172 \\
& 200 & 0.2368 & 0.1497 & 0.7250 & 0.3475 \\
$1,2,3,0.5$ & 400 & 0.2595 & 0.1790 & 0.5500 & 0.3451 \\
& & & & & \\
& 25 & 0.2595 & 0.1790 & 0.5500 & 0.3451 \\
& 50 & 1.2681 & 1.0026 & 1.5595 & 0.9923 \\
& 100 & 0.9265 & 0.5212 & 1.4801 & 0.9860 \\
$1,3,3,1$ & 200 & 0.5414 & 0.5895 & 0.5180 & 0.2900 \\
& 400 & 0.3024 & 0.2383 & 0.5288 & 0.3855 \\
& & & & & \\
& 25 & 0.7468 & 1.7881 & 1.8708 & 0.6623 \\
& 50 & 0.8613 & 1.1906 & 1.7275 & 0.6884 \\
& 100 & 0.7810 & 1.4113 & 1.2256 & 0.7246 \\
& 200 & 0.6085 & 0.7819 & 0.9493 & 0.5616 \\
& 400 & 0.6610 & 0.7129 & 0.8542 & 0.6231 \\
\hline
\end{tabular}




\section{Application}

In this section, we consider two real data sets to illustrate the applicability of the TGG distribution. The first data have been obtained from Nicholas and Padgett (2006) represents the tensile strength of 100 observations of carbon fibers observations.

Table 3. MLEs of the unknown Parameters for carbon fibers data with their corresponding SE in parenthesis, KS-Test, Cramér-von Mises and Anderson-Darling goodness of-fit statistics

\begin{tabular}{|c|c|c|c|c|c|c|c|}
\hline \multirow[t]{2}{*}{ Distribution } & \multicolumn{4}{|c|}{ Parameter Estimates } & \multirow[t]{2}{*}{$\mathrm{K}-\mathrm{S}$ test } & \multirow[t]{2}{*}{$\mathcal{W}$} & \multirow[t]{2}{*}{$\mathcal{A}$} \\
\hline & $\widehat{\alpha}$ & $\hat{\beta}$ & $\hat{\eta}$ & $\hat{\lambda}$ & & & \\
\hline TGG & $\begin{array}{c}0.2689 \\
(0.1174)\end{array}$ & $\begin{array}{c}2.9105 \\
(0.8954)\end{array}$ & $\begin{array}{c}0.3949 \\
(0.1451)\end{array}$ & $\begin{array}{c}0.6816 \\
(0.3462)\end{array}$ & 0.0612 & 0.0701 & 0.4107 \\
\hline GG & $\begin{array}{c}0.4230 \\
(0.1486)\end{array}$ & $\begin{array}{c}3.4520 \\
(1.0933)\end{array}$ & $\begin{array}{c}0.3360 \\
(0.1160)\end{array}$ & - & 0.0641 & 0.0789 & 0.4616 \\
\hline TG & $\begin{array}{c}0.0381 \\
(0.0097)\end{array}$ & - & $\begin{array}{c}0.8996 \\
(0.0842)\end{array}$ & $\begin{array}{c}0.7354 \\
(0.1718)\end{array}$ & 0.0860 & 0.1400 & 1.1239 \\
\hline GE & $\begin{array}{c}7.8778 \\
(1.5167)\end{array}$ & - & $\begin{array}{c}1.0211 \\
(0.0880)\end{array}$ & - & 0.1108 & 0.2318 & 1.2028 \\
\hline RE & $\begin{array}{c}1.19 \mathrm{e}+03 \\
(1.02 \mathrm{e}+03)\end{array}$ & - & $\begin{array}{c}1.05 \mathrm{e}-02 \\
(4.57 \mathrm{e}-03)\end{array}$ & - & 0.1438 & 0.0893 & 0.4842 \\
\hline G & $\begin{array}{c}0.0781 \\
(0.0176)\end{array}$ & - & $\begin{array}{c}0.7886 \\
(0.0773)\end{array}$ & - & 0.1025 & 0.1768 & 1.3637 \\
\hline
\end{tabular}

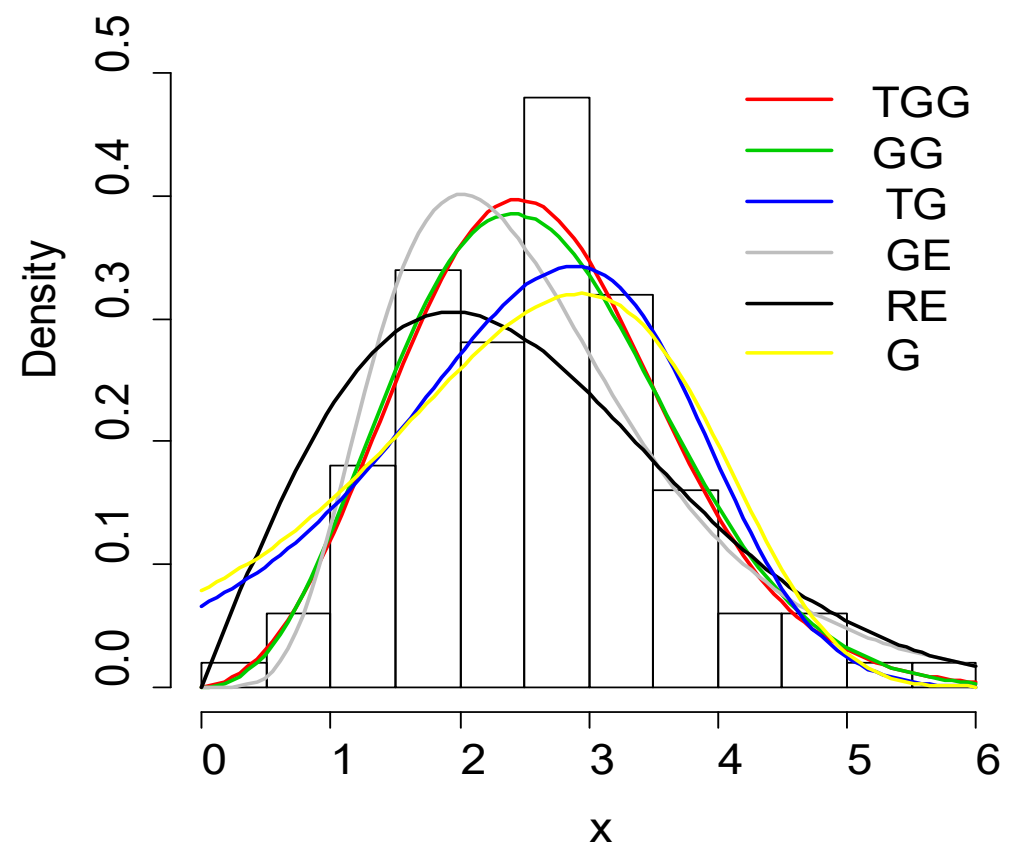

Fig. 4. Plots of the estimated densities of the TGG, GG, TG, GE, RE and G models. 
To illustrate the usefulness of the transmuted generalized Gompertz (TGG) distribution for the breaking stress of carbon fibers data, we compare its goodness of fit with sub-models, namely the generalized Gompertz (GG), transmuted Gompertz (TG), generalized exponential (GE), Rayleigh exponential (RE) and Gompertz (G) distributions. Maximum likelihood estimation was implemented by using BFGS method to minimize the $\log$ likelihood function in R. The MLEs of the TGG, GG, TG, GE, RE and G distributions with their corresponding standard errors, Kolmogorov-Smirnov (K-S) test, Cramér-von Mises and Anderson-Darling goodness of-fit statistics measures are displayed in Table 3. From Table 3, the smallest the values of these statistics indicate the better fit, which implies that the TGG distribution provides the better fit than the other five distributions.

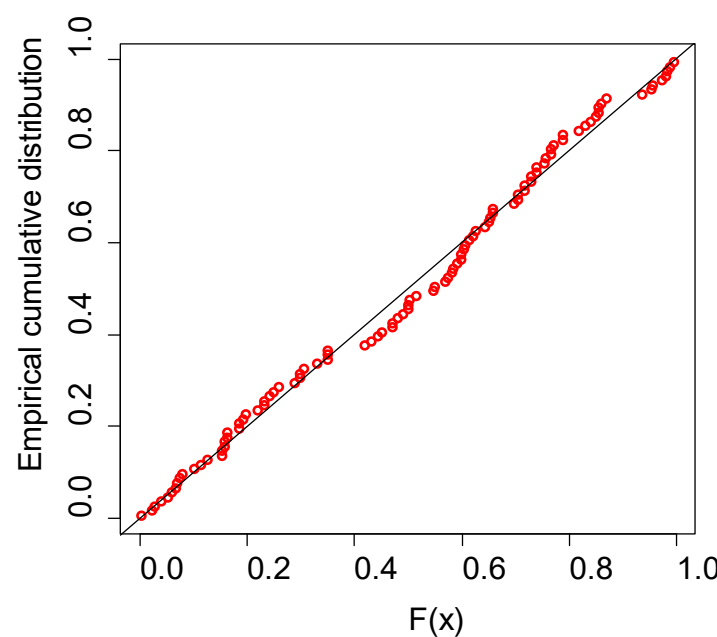

(a) TGG

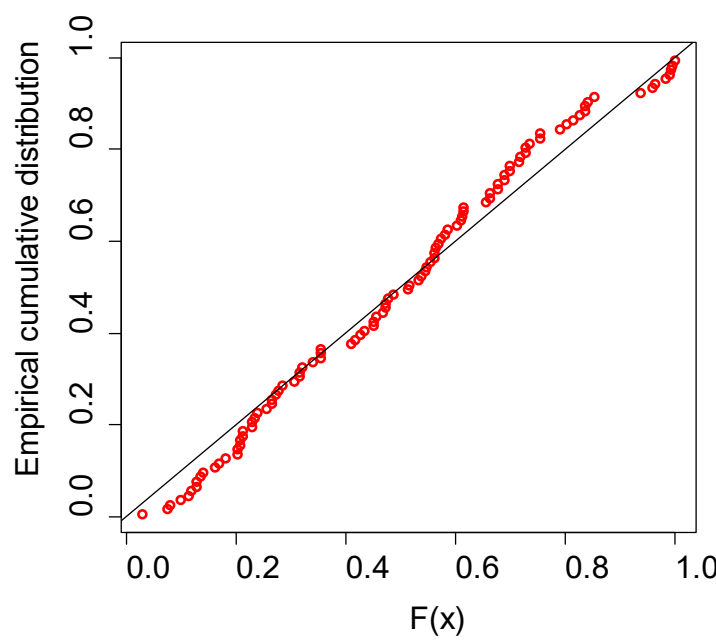

(c) TG

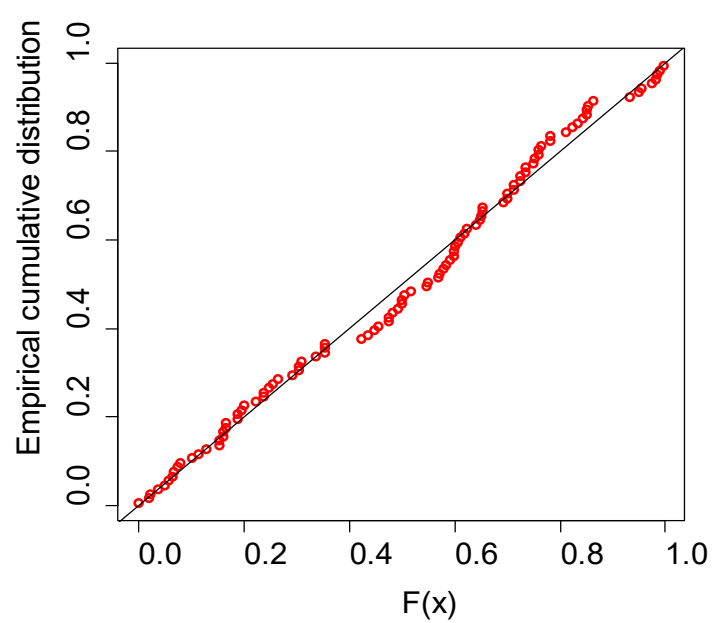

(b) GG

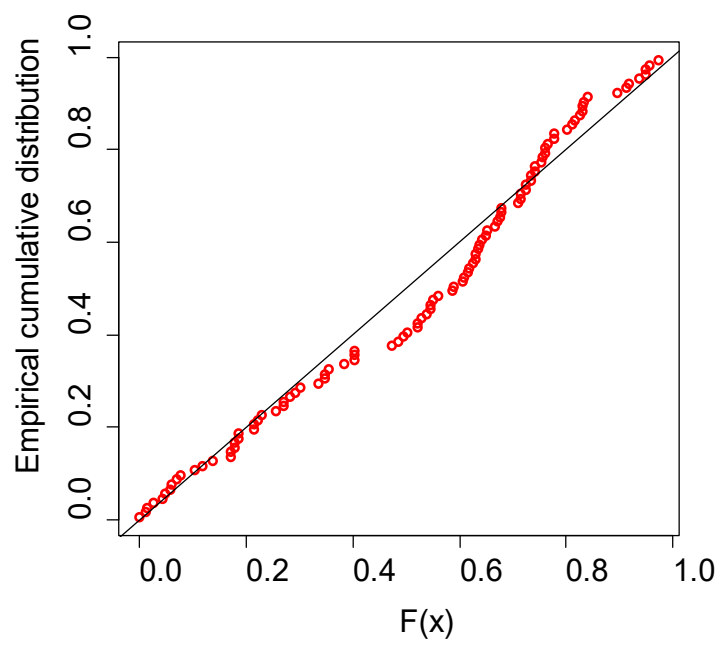

(d) GE 


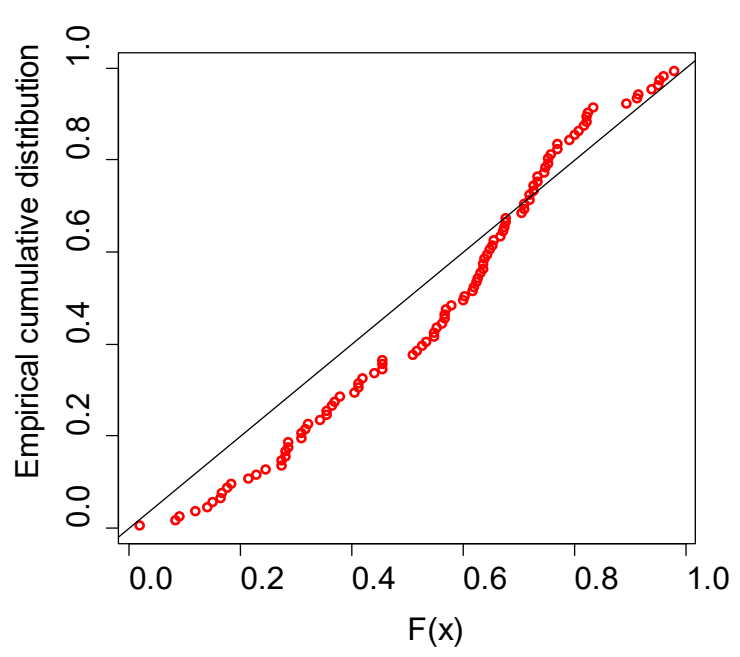

(e) $\mathrm{RE}$

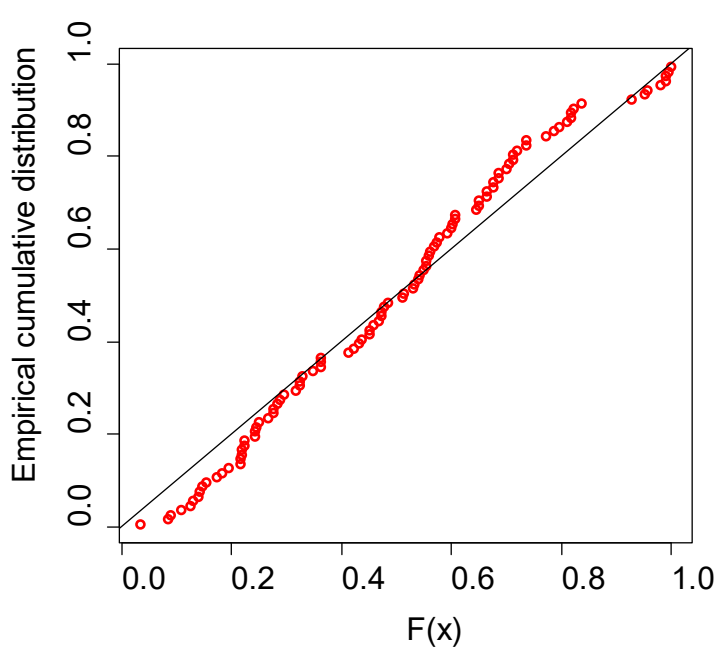

(f) $\mathrm{G}$

Fig. 5. P-P Plots of the TGG, GG, TG, GE, RE and G models for carbon fibers data.

Plots of the fitted densities for the TGG, GG, TG, GE, RE and G distributions for the carbon fibers data are displayed in Figure 4. We also display the pp-plots to decide which model provides the better fit for the carbon fibers data. Figure 5 shows the pp-plots of the six fitted models. From Figure 5, we observe that the TGG distribution fits the carbon fibers data well as the patterns of the points are very close to the 45-degree line (ab-line). Based on the goodness of fit measures, we conclude that the TGG distribution appears to be more appropriate than the five other lifetime distributions for carbon fibers data.

Table 4. MLEs of the unknown Parameters for uncensored carbon data with their corresponding SE in parenthesis, KS-Test, Cramér-von Mises and Anderson-Darling goodness of-fit statistics

\begin{tabular}{|c|c|c|c|c|c|c|c|}
\hline \multirow[t]{2}{*}{ Distribution } & \multicolumn{4}{|c|}{ Parameter Estimates } & \multirow[t]{2}{*}{ K-S test } & \multirow[t]{2}{*}{$\mathcal{W}$} & \multirow[t]{2}{*}{$\mathcal{A}$} \\
\hline & $\hat{\alpha}$ & $\hat{\beta}$ & $\hat{\eta}$ & $\hat{\lambda}$ & & & \\
\hline TGG & $\begin{array}{c}0.0897 \\
(0.0684)\end{array}$ & $\begin{array}{c}1.9756 \\
(0.7472)\end{array}$ & $\begin{array}{c}0.7682 \\
(0.2284)\end{array}$ & $\begin{array}{c}0.6831 \\
(0.3249)\end{array}$ & 0.0741 & 0.0624 & 0.3881 \\
\hline GG & $\begin{array}{c}0.1646 \\
(0.0967)\end{array}$ & $\begin{array}{c}2.2942 \\
(0.8691)\end{array}$ & $\begin{array}{c}0.6706 \\
(0.1807)\end{array}$ & - & 0.0832 & 0.0763 & 0.4677 \\
\hline TG & $\begin{array}{c}0.0176 \\
(0.0065)\end{array}$ & - & $\begin{array}{c}1.1934 \\
(0.1222)\end{array}$ & $\begin{array}{c}0.7096 \\
(0.2265)\end{array}$ & 0.0945 & 0.0820 & 0.6021 \\
\hline GE & $\begin{array}{c}9.1991 \\
(2.1491)\end{array}$ & - & $\begin{array}{c}1.0075 \\
(0.1001)\end{array}$ & - & 0.1549 & 0.3472 & 1.9082 \\
\hline $\mathrm{RE}$ & $\begin{array}{c}1.44 \mathrm{e}+03 \\
(1.56 \mathrm{e}+03)\end{array}$ & - & $\begin{array}{c}9.22 \mathrm{e}-03 \\
(5.05 \mathrm{e}-03)\end{array}$ & - & 0.2292 & 0.1595 & 0.8525 \\
\hline G & $\begin{array}{c}0.0373 \\
(0.0125)\end{array}$ & - & $\begin{array}{c}1.0701 \\
(0.1132)\end{array}$ & - & 0.1118 & 0.1099 & 0.7843 \\
\hline
\end{tabular}

The second data set represents the uncensored real data set on the breaking stress of carbon (in Gba) recently studied by Cordeiro et al. (2014). The data set describes the 66 observations are 3.70, 2.74, 2.73, 2.50, 3.60, $3.11,3.27,2.87,1.47,3.11,3.56,4.42,2.41,3.19,3.22,1.69,3.28,3.09,1.87,3.15,4.90,1.57,2.67,2.93$, 
$3.22,3.39,2.81,4.20,3.33,2.55,3.31,3.31,2.85,1.25,4.38,1.84,0.39,3.68,2.48,0.85,1.61,2.79,4.70$, $2.03,1.89,2.88,2.82,2.05,3.65,3.75,2.43,2.95,2.97,3.39,2.96,2.35,2.55,2.59,2.03,1.61,2.12,3.15$, $1.08,2.56,1.80,2.53$. We obtained the MLEs of the TGG, GG, TG, GE, RE and G distributions for the uncensored carbon data. The goodness of fit statistics including the Kolmogorov-Smirnov (K-S) test, Cramérvon Mises and Anderson-Darling goodness of-fit statistics measures to compare the fitted models. Table 4 listed the MLEs of the fitted models with their corresponding standard errors in parenthesis and three goodness of-fit statistics measures for data set 2. Based on the goodness of-fit statistics measures we have supporting evidence that the TGG distribution provides the better fit for the data set 2 .

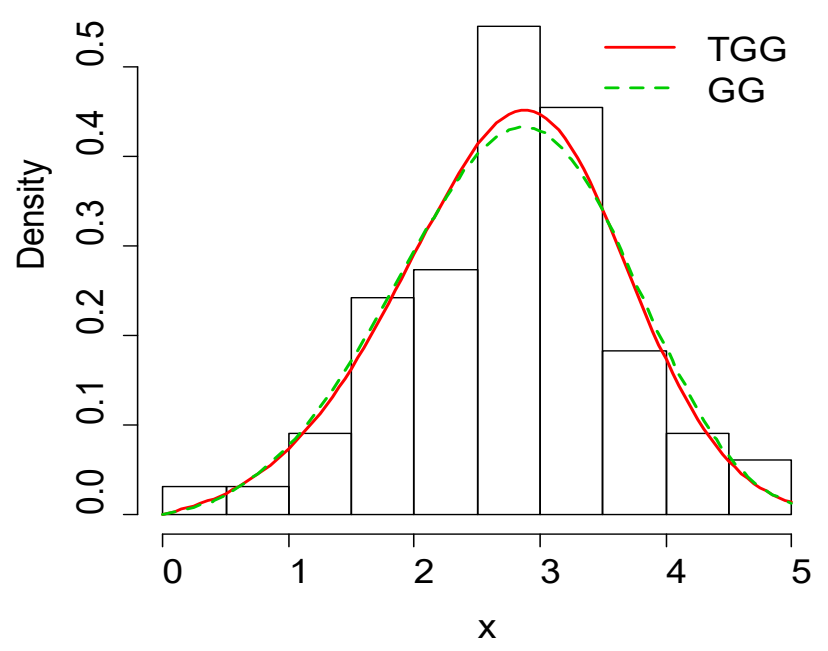

Fig. 6. Plots of the estimated densities of TGG and GG models for uncensored carbon data

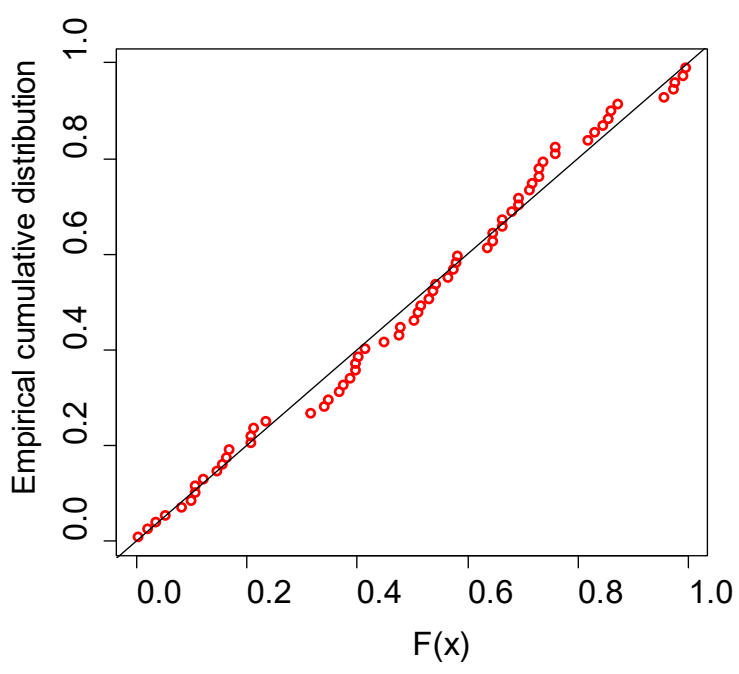

(a) P-P Plot

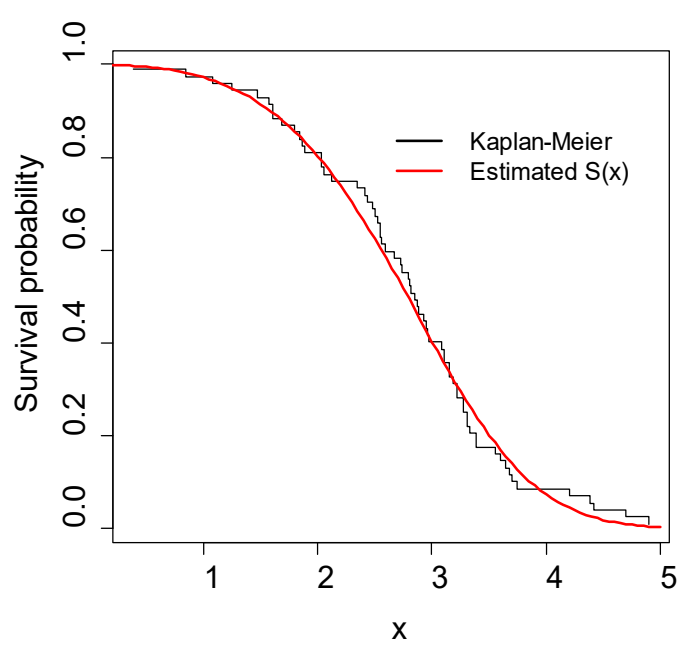

(b) Estimated Survival function

Fig. 7. P-P Plot and estimated survival of the TGG model for uncensored carbon data. 
Plots of the fitted densities for the TGG and GG distributions for the uncensored carbon fibers data are displayed in Figure 6. P-P Plot and the plots of the empirical survival function with the fitted survival function are presented in Figure 7. The points in the pp-plot are very close to the ab-line for the fitted TGG model. Furthermore the empirical and fitted survival function recommends that the TGG distribution provides the better fit for the uncensored carbon data.

\section{Concluding Remarks}

In this research we propose the four parameter transmuted generalized Gompertz distribution by using the quadratic rank transmutation map procedure proposed by Shaw and Buckley (2007). We investigate some of its mathematical properties such quantile function, moments, moment generating function, Rényi and $q$ entropies. Maximum likelihood estimation is proposed for estimating the model parameters. Finally two real data sets was analysed to show the potential usefulness of the TGG model. Based on the goodness of fit measures, we select the TGG model as an adequate model for fitting lifetime data. This is also justified by the estimated densities, empirical and fitted survival function and the pp-plots.

\section{References}

[1] Aryal, G R. and Tsokos, C P. (2011) Transmuted Weibull distribution: A Generalization of the Weibull Probability Distribution. European Journal of Pure and Applied Mathematics, Vol. 4, No. 2, 89-102.

[2] Bowley, A. L. (1920). Elements of statistics. C. Scribner's sons, New York.

[3] Bourguignon, M., Ghosh, I., and Cordeiro, G. M. (2016). General results for the transmuted family of distributions and new models, Journal of Probability and Statistics, Vol. 2016, Article ID 7208425, 12 pages, http://dx.doi.org/10.1155/2016/7208425.

[4] Cordeiro G. M, Ortega Edwin M.M. and. Lemonte Artur. J. (2014) The exponential-Weibull lifetime distribution, Journal of Statistical Computation and Simulation, Vol. 84, No. 12, 2592-2606.

[5] Gompertz, B. (1825). On the Nature of the Function Expressive of the Law of Human Mortality and on a New Mode of Determining the Value of Life Contingencies. Philosophical Transactions of the Royal Society of London, Vol. 115, 513-583.

[6] Garg M.L., Rao B.R., Redmond C.K., (1970). Maximum likelihood estimation of the parameters of the Gompertz survival function, Journal of the Royal Statistical Society C, Vol. 19, 152-159.

[7] Gohary A. El, Alshamrani Ahmad, Otaibi .Adel Naif Al, (2013). The generalized Gompertz distribution, Applied Mathematical Modelling, Vol. 37, 13-24.

[8] Havrda, J. and Charvat, F. (1967). Quantification method in classification processes: concept of structural $\alpha$-entropy. Kybernetika, Vol. 3, 30-35.

[9] Khan M. Shuaib, King Robert. (2013a). Transmuted Modified Weibull Distribution: A Generalization of the Modified Weibull Probability Distribution, European Journal of Pure and Applied Mathematics, Vol. 6: 1, 66-88.

[10] Khan M. Shuaib and King Robert. (2013b). Transmuted generalized Inverse Weibull distribution, Journal of Applied Statistical Sciences, Vol. 20: 3, 15-32.

[11] Khan M. Shuaib, King Robert and Hudson Irene. (2014a). Characterizations of the transmuted Inverse Weibull distribution, ANZIAM J. Vol. 55 (EMAC2013), C197-C217.

[12] Khan M. Shuaib and King Robert. (2014b). A New Class of Transmuted Inverse Weibull Distribution for Reliability Analysis, American Journal of Mathematical and Management Sciences, Vol. 33, No.4, 261-286.

[13] Khan, M. Shuaib, King Robert, Hudson Irene. (2014c). Transmuted Gompertz distribution with application" presented in the 8th Australia - New Zealand Mathematics Convention, held at Melbourne at Parkville Campus the University of Melbourne, from December 8-12, 2014.

[14] Khan, M. Shuaib, King Robert, Hudson Irene. (2015). A new three parameter transmuted Chen lifetime Distribution with application, Journal of Applied Statistical Sciences, Vol. 21, No. 3, 239-259.

[15] Moniem. Abdul. I. B; Seham. M. (2015). Transmuted Gompertz Distribution, Computational and Applied Mathematics, Vol. 1 , No. 3, 88-96.

[16] Merovci, F. (2013). Transmuted Rayleigh distribution. Austrian Journal of Statistics, Vol. 42, No. 1, 21-31.

[17] Nicholas M. D. and Padgett W. J, (2006). A bootstrap control chart for Weibull percentiles, Quality and Reliability Engineering International. Vol. 22, 141-151.

[18] Rényi, A. (1961). On measures of entropy and information. University of California Press, Berkeley, California, 547-561. 
[19] R Development Core Team, (2013). A Language and Environment for Statistical Computing, R Foundation for Statistical Computing. Vienna, Austria.

[20] Shaw. W. and Buckley. I. (2007). The alchemy of probability distributions: beyond Gram-Charlier expansions and a skewkurtotic-normal distribution from a rank transmutation map. Research report, King's College, London, U.K.

[21] Ullah, A. (1996). Entropy, divergence and distance measures with econometric applications. Journal of Statistical Planning and Inference. Vol. 49, 137-162.

[22] Yuzhu Tian, Maozai Tian \& Qianqian Zhu. (2014). Transmuted Linear Exponential Distribution: A New Generalization of the Linear Exponential Distribution, Communications in Statistics - Simulation and Computation, Vol. 43, No.10, 2661-2677 